\title{
Coumarin, a Lead Compound of Warfarin, Inhibits Melanogenesis via Blocking Adenylyl Cyclase
}

\author{
Dong-Chan Kim ${ }^{1, *}$, Seong-Hwan Rho ${ }^{2}$, Dongjin Kim ${ }^{3}$, Sung In Kim ${ }^{1}$, Chul-Soo Jang ${ }^{1}$, Jae Ki Ryu ${ }^{1}$, \\ Byung Weon Kim ${ }^{1}$, Chang Oh Kweon ${ }^{1}$, Hyun-kyung Kim ${ }^{1}$, Suk Jun Lee ${ }^{1}$ \\ ${ }^{1}$ Department of Biomedical Laboratory Science, Gimcheon University, Gimcheon City, South Korea \\ ${ }^{2}$ Institute for Physics, University of Freiburg, Freiburg, Germany \\ ${ }^{3}$ School of Civil and Environmental Engineering, Georgia Institute of Technology, Atlanta, U.S.A \\ *Corresponding author: jenokin@nate.com
}

Received April 16, 2013; Revised April 26, 2013; Accepted April 28, 2013

\begin{abstract}
Due to Its multiple biological activities, coumarin (a main ingredient of Cinnamon extracts) has gained attention as potentially useful therapeutics for various diseases. However, the efficacy of coumarin for the use of dermatological health has not been fully explored. To clarify the action mechanism of the skin protecting property of coumarin, we firstly investigated the molecular docking property of coumarin on the mammalian adenylyl cyclase, which is the key enzyme of cAMP-induced melanogenesis in the skin cells. In binding study, the benzopyran moiety of coumarin occupies dual sites of the hydrophobic cleft at the interface of two subunits of adenylyl cyclase. We also examined the involvement of coumarin in alpha-MSH and forskolin induced cAMP signaling within a cell based assay. In addition, we inquired into the inhibitory effect of coumarin on melanogenesis and found that the pretreatment with coumarin inhibited the forskolin-induced melanin contents significantly without annihilating the cell viability. Our results strongly suggest that coumarin directly inhibits the activity of adenylyl cyclase, downregulates forskolin-induced cAMP-production pathway, consequently inhibiting melanogenesis. Thus, coumarin may also be used as an effective inhibitor of hyperpigmentation.
\end{abstract}

Keywords: coumarin, melanin production inhibitor, cyclic AMP

\section{Introduction}

Skin-whitening is big cosmetic business in countries like South Korea, India, China, and Taiwan where many women view whiter skin as a symbol of good health, high social status and beauty. Thus, drug discovery in skin pharmacotherapy is an enormous, continually expanding field. Melanin is main target for skin pharmacotherapy, and is synthesized in special organelles called melanosomes that play an essential role in protecting skin from deleterious sunlight under normal conditions [1]. However, enhanced generation and excessive accumulation of melanin can cause a number of skin problems such as freckles, age spots, and melasma [2]. Melanin synthesis is stimulated by a large number of effectors, including 1-oleyl-2-acetyl-glycerol [3], ultraviolet $\mathrm{B}$ radiations [4], and cAMP-elevating agents (forskolin, $\alpha$ MSH, IBMX) [5]. The major signaling pathway leading to melanin synthesis appears to be the stimulation of adenylyl cyclase followed by an increase in the cAMP level and the activation of cAMP-dependent protein kinases (PKAs). cAMP increases the expression of the micropthalmia-associated transcription factor (MITF), a melanocyte-specific transcription factor crucial for melanocyte development and differentiation, through activation of the cAMP-dependent protein kinase A (PKA) and the cAMP-response element binding protein (CREB) transcription factor, which in turn stimulates tyrosinase gene expression to allow melanin synthesis [6]. Therefore, the inhibitors of the melanogenesis via blocking adenylyl cyclase activity and its signaling pathway, particularly from natural sources, can be a great interest to industries that produce skin whitening and cosmetics.

Adenylyl cyclase (AC) is a 12-transmembrane protein that converts ATP to cAMP upon the stimulation of various G-protein coupled receptors [7]. In Eukaryotes, two basic families of $\mathrm{AC}$ exist: the $\mathrm{G}$ protein-regulated transmembrane AC isoforms and the soluble AC. The soluble AC is regulated by bicarbonate and calcium and is insensitive to forskolin or activated $\mathrm{G} \alpha_{\mathrm{s}}$ [8]. The topology of transmembrane ACs consists of a variable in tracellular $\mathrm{N}$ terminus and two large cytoplasmic domains separated by two membrane-spanning domains (six transmembranes each) [9]. The transmembrane class is generally considered the target of most hormone-sensitive cAMP control. So far nine mammalian AC isoforms have been identified. These isoforms show distinct tissue distribution and biological/pharmacological properties, while they share the same membrane topology and catalytic core formation that resembles transporters [7]. All nine membrane-bound AC isoforms are activated by GTPbound $\mathrm{G \alpha}_{\mathrm{s}}$ and the plant diterpene forskolin, with the exception of AC9. Forskolin, a direct activator of AC, is a diterpene extract from coleus forskolii, which is a natural plant used in Indian traditional medicine for the treatment 
of heart failure, bronchial asthma, and alimentary diseases [10].

Coumarin (2H-chromen-2-one, benzo- $\alpha$-pyrone) and its derivatives are widely distributed in plants and are especially abundant in the bark and leaves of plants $[11,12]$. In addition, coumarin is a fragrant chemical compound of benzopyrone notably found in cinnamon extracts $[13,14,15]$. Coumarins have shown evidence of many biological activities. The biomedical activities of coumarin includes anti-HIV [16], anti-inflammatory [17], anti-cancer [18], and anti-diabetes [19]. Despite of coumarin's many pharmaceutical effects, the skin protective function of coumarin has not been fully studied. Therefore, by using the well-characterized Autodock 4.2 and Autodock Vina 1 computational modeling system and B16 mouse melanoma cell based systems, we attempted to determine the skin pharmaceutical effects and molecular binding mechanisms of coumarin on adenylyl cyclase and melanogenesis. In this investigation, we first demonstrated that coumarin effectively inhibits melanogenesis via dual blocking adenylyl cyclase activity and directly affects on cAMP production in B16 melanocytes.

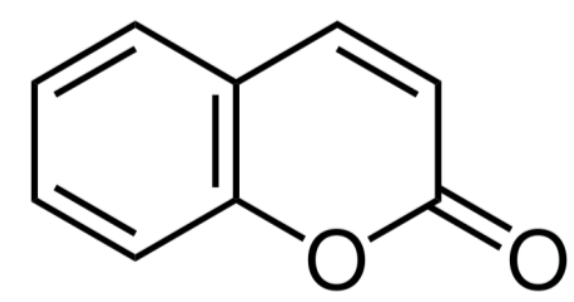

Figure 1. Chemical structure of $2 \mathrm{H}$-chromen-2-one (coumarin)

\section{Materials and Methods}

\subsection{Reagents}

Coumarin, forskolin, $\alpha-\mathrm{MSH}$, MTT and other chemical reagents were purchased from Sigma Chemical Co. (St. Louis, MO, U.S.A.). $\left[{ }^{3} \mathrm{H}\right]$ cAMP was purchased from PerkinElmer Life and Analytical Sciences (Boston, MA).

\subsection{Molecular Docking}

AutoDock 4.2 [20] and AutoDock Vina 1 [21] were used for the docking study of coumarin. The crystallographic structures of two catalytic subunits of mAC, VC1 and IIC2 as well as that of forskolin, a known ligand of $\mathrm{mAC}$, were adopted from the PDB file $1 \mathrm{UOH}$ [22]. To prepare ligands and the receptor molecule for docking, ADT [23] was used to add hydrogens and calculate atomic charges. Coumarin has zero rotatable bonds. Ten amino acid residues at the docking site in the receptor molecule were assumed to be flexible; Leu A438, Asp A440, Thr A512, Asn A515, Lys B938, Ile B940, Ser B942, Asp B1018, Asn B1025, and Lys B1065. A search box was defined to contain both binding sites of forskolin and 2 '(3')-O-(N-methylanthraniloyl) (MANT)-GTP (37.5 $\left.\times 24.0 \times 27.0 \AA^{3}\right)$. AutoDock Vina uses a grid spacing of 1 $\AA$ and Autodock 4.2 uses $0.375 \AA$ by default. The docking computation was performed on a 64bit Linux PC with a $3 \mathrm{GHz}, 8$ multi-threaded Intel CPU and 16 GB RAM. The result was analyzed and visualized by the software PyMOL [24].

\subsection{Cell Culture}

B16 cells were purchased from Korean Cell Line Bank (Seoul, Korea) were cultured in DMEM with $10 \%$ fetal bovine serum (FBS; Gibco, NY, U.S.A.) and penicillin/streptomycin $(100 \mathrm{IU} / 50 \mu \mathrm{g} / \mathrm{ml})$ in a humidified atmosphere containing $5 \% \mathrm{CO}_{2}$ in air at $37^{\circ} \mathrm{C}$. B16 cells were cultured in 24-well plates for each assay. All the experiments were performed in triplicate and repeated three times to ensure reproducibility.

\subsection{Measurement of $\left[{ }^{3} \mathrm{H}\right]$ cAMP}

Intracellular cyclic AMP generation was determined by $\left[{ }^{3} \mathrm{H}\right]$-cyclic AMP competition assay in binding to cyclic AMP binding protein as described previously by [25] with some modification. To determine the cyclic AMP production induced by $\alpha-\mathrm{MSH}$ and forskolin, the B16 cells were stimulated with agonists for $20 \mathrm{~min}$ in the presence of the phosphodiesterase inhibitor Ro 20-1724 (5 $\mu \mathrm{M})$ and the reaction was quickly terminated by three repeated cycles of freezing and thawing. The samples were then centrifuged at $2500 \times \mathrm{g}$ for $5 \mathrm{~min}$ at $4^{\circ} \mathrm{C}$. The cyclic AMP assay is based on the competition between $\left[{ }^{3} \mathrm{H}\right]$-labelled cyclic AMP and unlabelled cyclic AMP present in the sample for binding to a crude cyclic AMP-binding protein prepared from bovine adrenal cortex according to the method of [26]. Each sample was incubated with $50 \mu$ l of $\left[{ }^{3} \mathrm{H}\right]$-labelled cyclic AMP $(5 \mathrm{mCi})$ and $100 \mu \mathrm{l}$ of binding protein for $2 \mathrm{~h}$ at $4^{\circ} \mathrm{C}$. Separation of the protein-bound cyclic AMP from the unbound cyclic AMP was achieved by the adsorption of the free cyclic AMP onto charcoal $(100 \mu \mathrm{l})$ followed by centrifugation at $12,000 \times g$ at $4^{\circ} \mathrm{C}$. The $200 \mu \mathrm{l}$ of supernatant were then placed into an Eppendorf tube containing $1.2 \mathrm{ml}$ of scintillation cocktail to measure the radioactivity. The cyclic AMP concentration in the sample was determined based on a standard curve and expressed as pmol per number of cells.

\subsection{Estimation of Melanogenesis in B16 Cells}

Melanin content was used as an index of melanogenesis. Determination of melanin content was performed using a modified method of previous study [27]. In brief, B16 cells $\left(5 \times 10^{4}\right)$ were plated on 24-well, multi-dishes and pretreated with coumarin at concentrations ranging from 0 to $300 \mu \mathrm{M}$. Then added and incubated with $100 \mathrm{nM}$ of forskolin for $24 \mathrm{hrs}$. After washing twice with PBS, samples were dissolved in $100 \mathrm{mll}$ of $1 \mathrm{~N} \mathrm{NaOH}$. The samples were incubated at $60^{\circ} \mathrm{C}$ for $1 \mathrm{hr}$ and mixed to solubilize the melanin. Absorbance at $405 \mathrm{~nm}$ was compared with a standard curve of synthetic melanin.

\subsection{Determination of Chrysin for Cell Viability}

The cell viability assay was performed as described in the previous study [28] using 3-(4,5-dimethylthiazol-2yl)-2,5-diphenyltetrazolium bromide (MTT). Shortly thereafter, $5 \times 10^{4}$ B16 cells/well were plated in a 24 -well plate. After cells were exposed to coumarin at various time scales, MTT solutions were added and the insoluble 
derivative formed by cellular dehydrogenase was solubilized with EtOH-dimethyl sulfoxide (DMSO) (1:1 mixture solution); the absorbance of each well was read at $560 \mathrm{~nm}$ using a microplate spectrophotometer.

\subsection{Statistical Analysis}

The values are expressed as mean \pm standard error $(n=$ 3 ) and the biological significance $p<0.05$ was determined by the Student's $t$-test.

\section{Results}

\subsection{Molecular Docking of Coumarin}

Coumarin is a small and planar compound with the molecular weight of 146.04. This suggests that the docking of coumarin to two relatively large binding clefts of mAC might be a hard optimization problem. The docking solutions obtained from initial trials of coumarin to mAC with AutoDock 4.2 showed poor separations among the clusters of docked conformations. In total, 41 distinct conformational clusters were found out of 256 lamarckian genetic algorithm docking runs using an RMSD tolerance of $2.0 \AA$. The difference in mean binding energy between the top and the second clusters was $0.30 \mathrm{kcal} / \mathrm{mol}$. Therefore, care must be taken in adopting the top solution right away.

An additional independent docking calculation was performed with AutoDock Vina which adopted a stochastic global optimization algorithm in addition to a modified scoring function. It is shown that Vina has a much improved speed and accuracy in predictions when compared to AutoDock 4 [21]. As a control experiment, docking of forskolin, the known ligand of mAC was performed using Vina. In 10 out of 10 docking runs with a different random seed, the crystallographic binding mode was successfully found with an average RMSD of $0.6 \AA$, which is significant improvement when compared with $1.1 \AA$ from the same experiment in our previous report using AutoDock 4 [29].

For the docking study of coumarin, 20 independent docking runs using Vina were performed with different random seeds. As with AutoDock 4, the separation of the top cluster from the second cluster was only $0.3 \mathrm{kcal} / \mathrm{mol}$ in average, which again suggests the existence of local minima in the energy landscape and, thus, several possible binding modes. Interestingly, the best solutions of 20 runs are distributed in two distinct binding sites. In 16 out of 20 runs, the best docking cluster of coumarin was found at the forskolin binding site with the minimal binding energy of $-6.6 \mathrm{kcal} / \mathrm{mol}$ and in the remaining four runs, the best docking cluster was found at the MANT-GTP binding site with the minimal binding energy of $-6.4 \mathrm{kcal} / \mathrm{mol}$ (Figure 2. panels A). The existence of two binding modes was also observed with the docking runs using AutoDock 4. In this case, the best cluster composed of 19 runs out of 256 runs was found at the MANT-GTP binding pocket, while the second best cluster composed of 37 runs was found at the forskolin binding pocket.

In both possible binding modes, the benzopyran moiety of coumarin occupies the hydrophobic cleft at the interface of two subunits of mAC. The orientation of coumarin seems to be guided by hydrogen bonding between the carbonyl group of coumarin and the nearby hydrogen donor, which is Ser B942 or Asn B1025, respectively (Figure 2. panel B and C).

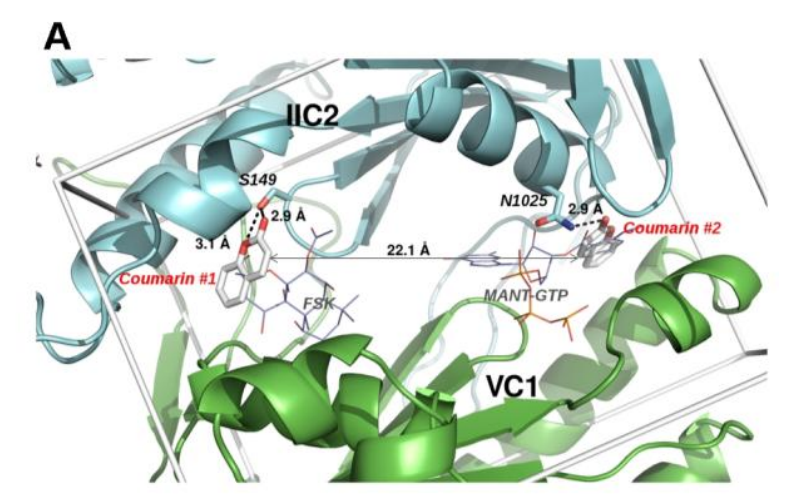

B

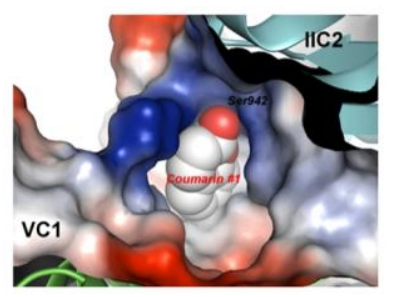

C

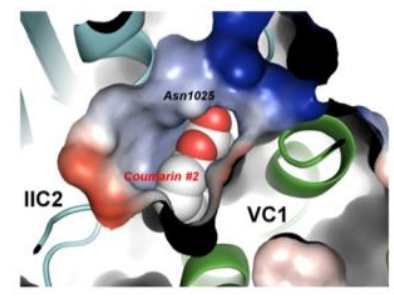

Figure 2. The predicted binding mode of coumarin to mAC. (A). Two best docking solutions of coumarin (stick, white colored) are visualized on the mAC structure (ribbon). For comparison, the crystallographic conformations of forskolin (FSK) and MANT-GTP are also displayed (lines). (B and C). Each docked coumarin molecule is visualized with the electrostatic potential mapped on the surface of mAC. The docked molecules are viewed from the center of the binding pocket space. The calculation was done by PyMOL using AMBER99 vacuum electrostatic potential.

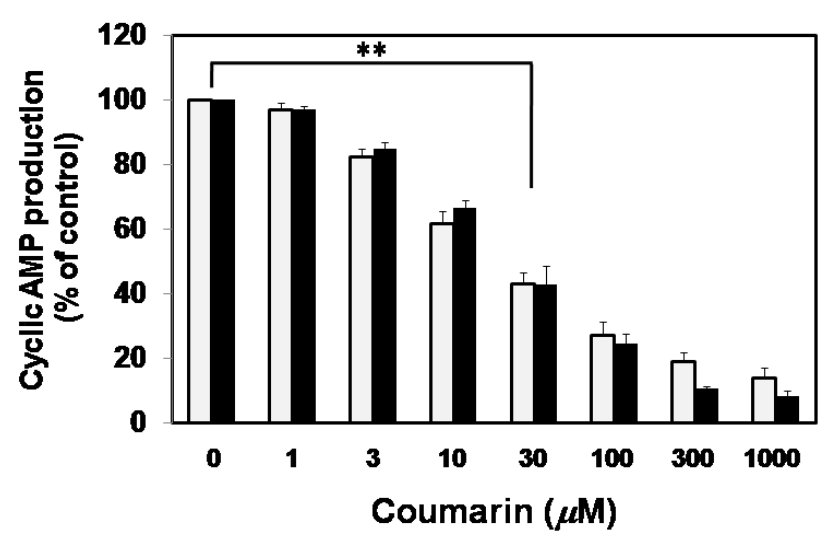

Figure 3. The inhibition of $\alpha$-MSH- and Forskolin- induced cAMP production with the increase in the concentration of coumarin treatment. B16 cells were preincubated with various concentrations of coumarin for $5 \mathrm{~min}$, then cells were stimulated with $\alpha-\mathrm{MSH}(100 \mathrm{nM}$, white bar) and forskolin(100nM, closed bar), respectively. The data are the mean \pm S.E.M. of assay triplicates with at least six independent experiments. $(* * \mathrm{p}<0.05)$.

\subsection{Inhibitory Action of Coumarin on Adenylyl Cyclase-Induced cAMP Production.}

To check whether coumarin really blocks adenylyl cyclase activity in living cellular system or not, we chose the B16 melanoma cell line and investigated the coumarin's inhibitory effect. Both $\alpha$-MSH and forskolin are known to increase intracellular cAMP concentration via activating adenylyl cyclase signaling pathways in B16 
cells [30]. We preliminarily checked the cAMP production induced by $\alpha-\mathrm{MSH}$ and forskolin in B16 cells (data not shown), and EC50 for both were 100nM. 5min pretreatment of coumarin showed strong inhibitory-effect on $\alpha-\mathrm{MSH}$ - and forskolin-induced cAMP production in a concentration-dependent manner (Figure 3) suggesting that coumarin inhibits cAMP production signaling pathways via blocking adenylyl cyclase.

\subsection{Inhibitory Action of Coumarin on Melanin Content in B16 Cells}

In order to obtain evidence for the inhibitory effect of coumarin on forskolin-induced melanogenesis signaling pathway in B16 cells, melanin production in B16 cells was examined. As shown in Figure $4 \mathrm{a}$, the melanin content of the cells that were pretreated with coumarin decreased in dose-dependent manners, showing $50.5 \%$ at $18 \mathrm{M}$ and $35.5 \%$ at $35 \mu \mathrm{M}$ compared to the forskolin alone treated control group. (a)

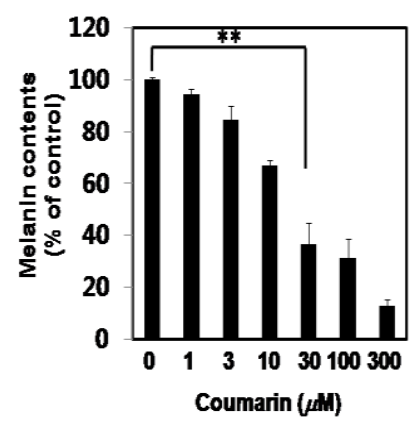

(b)

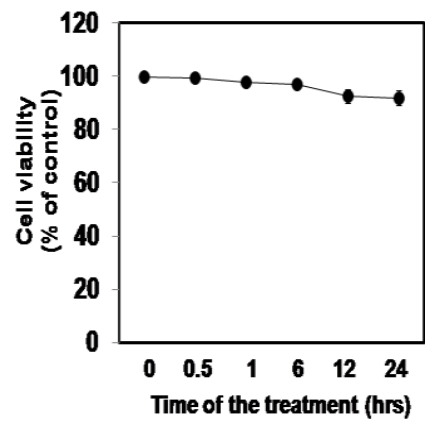

Figure 4. (a) The melanin content reduction with the increase in the concentration coumarin treatment on the melanin biosynthesis. The results are expressed as percentages of control, and the data are the mean \pm S.E.M. of at least three determinations. $(* * p<0.05)(b)$ The viability of B16 melanocytes during the coumarin treatments. Cells were treated over the indicated time (from $0 \mathrm{hrs}$ to $24 \mathrm{hrs}$ ) with $18 \mu \mathrm{M}$ of coumarin. The viability was evaluated by MTT assay and represented as percentages of control. The data are the mean \pm S.E.M. of assay triplicates.

\subsection{The Effect of Coumarin on Cell Viability}

The data from the cell viability assay using MTT for B 16 cells are shown in Figure 4b. At IC50 concentration of coumarin $(18 \mu \mathrm{M})$, the B16 cells were treated for $24 \mathrm{hrs}$. Long-term treatment of coumarin did not induce significant cellular death. These data clearly indicate that the anti-melanogenesis effect of coumarin is not due to its cytotoxicity.

\section{Discussion}

The result of docking study implies two binding sites of coumarin in $\mathrm{mAC}$, one at the forskolin binding pocket and the other at the MANT-GTP binding pocket. The difference of binding free energy between two binding modes was indistinguishable, considering the reported standard error in the binding energy calculation by Vina is $2.85 \mathrm{kcal} / \mathrm{mol}$ [21]. When compared to the best docking energy of forskolin, which is $-10.3 \mathrm{kcal} / \mathrm{mol}$, the obtained binding energy of coumarin is significantly larger. The calculated inhibition constant $\mathrm{K}_{\mathrm{i}}$ of forskolin and coumarin are $14.5 \mathrm{nM}$ and $28.2 \mu \mathrm{M}$, respectively. The calculated $\mathrm{K}_{\mathrm{i}}$ vaule of coumarin to $\mathrm{mAC}$ in the micromolar range is comparable to the $K_{i}$ values of coumarin to various other target enzymes registered at the Binding Database [31].

Although the competition between inhibitor and substrate is needed to infer $\mathrm{IC}_{50}$ from $\mathrm{K}_{\mathrm{i}}$, our cellular assay showed that the $\mathrm{IC}_{50}$ value of coumarin was also slightly larger than that of chrysin, which showed a comparable $\mathrm{K}_{\mathrm{i}}$ with forskolin previously [29]. Interestingly, the difference of $\mathrm{IC}_{50}$ between coumarin and chrysin was not as dramatic as in docking energy comparison. If coumarin can occupy two substrate binding pockets of $\mathrm{mAC}$ simultaneously and each binding interaction is independent and inhibitory to mAC activity, coumarin can be a more potent inhibitor than $\mathrm{K}_{\mathrm{i}}$ suggests. Roughly speaking, at the concentration of $\mathrm{K}_{\mathrm{i}}$, half of coumarin molecules are bound to $\mathrm{mAC}$, however, only one quarter of mAC molecule is free from coumarin and, therefore, is active. Existence of two possible binding sites would be an interesting hypothesis to test in the future study.

The two predicted binding modes follow a common binding mechanism observed in the crystal structure of cytochrome P450 2A6 [32], where coumarin binds to a compact, hydrophobic active site of the protein and Asn297 residue acts as a hydrogen bond donor to orient coumarin. However, mAC offers much larger possible binding surface to coumarin. The two found binding positions of coumarin are approximately $22 \AA$ away from each other, leaving the major part of the binding pocket open. Due to this larger open space, the flexibility of the interacting side chains becomes influential by creating many suboptimal binding modes and therefore contributes to make the optimization process in docking calculation difficult. Under the realistic cellular environment, the precise binding coordinates will be fixed by the actual conformation of these side chains. It will be also interesting to test if metabolic products or derivatives of coumarin utilizing the remaining space can be much potent $\mathrm{mAC}$ inhibitors.

Coumarin is a polyphenol compound and known as very powerful anti-oxidants that smell like either vanilla or freshly mown hay (it's what smells nice after clover is cut). Because of this lovely smell, it's often used in perfumes and fragrance oils. Plant derived polyphenolic compounds, as potentially safe and effective skin protecting agents, have attracted many researchers in cosmeceutical industries. In many previous studies $[33,34,35,36,37]$, polyphenolic hydroxyl compound may show skin protection and whitening activity because of its ability to donate an electron and/or chelate transition metals, such as copper or ferrous ions, thereby inhibit free radical reactions and diminish ROS generation. One of the interesting findings of our study is that the potency of coumarin is likely related to its dual efficacy on antimelanogenic and antioxidative activities. As shown in this study, coumarin inhibited melanogenesis induced by adenylyl cyclase activity and was able to decrease melanin content without showing any adverse effect on cell viability. These strong anti-melanogenic and skin protecting actions of coumarin are ascribed to its structural arrangement. 


\section{Conclusions}

Overall, the elucidation of the dual binding site of coumarin on adenylyl cyclase could provide a structural basis for the pharmacological modulation of ACs and clues for the treatment related incurable diseases. Coumarin may be considered as a potent therapeutic agent for skin hyperpigmentation such as melanosis.

\section{Competing Interests}

The authors declare they have no competing interests.

\section{Acknowledgements}

This work was supported by the National R\&D Support Program of Small \& Medium Business Administration (SMBA), South Korea (Project Code \#S1021757) and NEURONEX. Co. Ltd.

\section{References}

[1] Smit, N., J. Vicanova, and S. Pavel, The hunt for natural skin whitening agents. Int J Mol Sci, 2009. 10(12): p. 5326-49.

[2] Slominski, A., et al., Melanin pigmentation in mammalian skin and its hormonal regulation. Physiol Rev, 2004. 84(4): p. 1155228.

[3] Friedmann, P.S., F.E. Wren, and J.N. Matthews, Ultraviolet stimulated melanogenesis by human melanocytes is augmented by di-acyl glycerol but not TPA. J Cell Physiol, 1990. 142(2): p. 33441 .

[4] Chakraborty, A.K., et al., UV light and MSH receptors. Ann N Y Acad Sci, 1999. 885: p. 100-16.

[5] Bowers, R.R., A. Biboso, and O. Chavez, The role of alpha-MSH, its agonists, and C-AMP in in vitro avian melanocytes. Pigment Cell Res, 1997. 10(1-2): p. 41-5.

[6] Bertolotto, C., et al., Regulation of tyrosinase gene expression by cAMP in B16 melanoma cells involves two CATGTG motifs surrounding the TATA box: implication of the microphthalmia gene product. J Cell Biol, 1996. 134(3): p. 747-55.

[7] Dwivedi, Y. and G.N. Pandey, Adenylyl cyclase-cyclicAMP signaling in mood disorders: role of the crucial phosphorylating enzyme protein kinase A. Neuropsychiatr Dis Treat, 2008. 4(1): p. 161-76.

[8] Kamenetsky, M., et al., Molecular details of cAMP generation in mammalian cells: a tale of two systems. J Mol Biol, 2006. 362(4): p. 623-39.

[9] Sadana, R. and C.W. Dessauer, Physiological roles for G proteinregulated adenylyl cyclase isoforms: insights from knockout and overexpression studies. Neurosignals, 2009. 17(1): p. 5-22.

[10] Iwatsubo, K., T. Tsunematsu, and Y. Ishikawa, Isoform-specific regulation of adenylyl cyclase: a potential target in future pharmacotherapy. Expert Opin Ther Targets, 2003. 7(3): p. 441-51.

[11] Ngo, N.T., et al., Cytotoxic Coumarins from the Bark of Mammea siamensis. Chem Pharm Bull (Tokyo). 58(11): p. 1487-91.

[12] Akak, C.M., et al., New coumarin glycosides from the leaves of Diospyros crassiflora (Hiern). Fitoterapia. 81(7): p. 873-7.

[13] Rychlik, M., Quantification of free coumarin and its liberation from glucosylated precursors by stable isotope dilution assays based on liquid chromatography-tandem mass spectrometric detection. J Agric Food Chem, 2008. 56(3): p. 796-801.

[14] Woehrlin, F., et al., Quantification of flavoring constituents in cinnamon: high variation of coumarin in cassia bark from the
German retail market and in authentic samples from indonesia. $\mathbf{J}$ Agric Food Chem. 58(19): p. 10568-75.

[15] Blahova, J. and Z. Svobodova, Assessment of coumarin levels in ground cinnamon available in the Czech retail market. ScientificWorldJournal, 2012. 2012: p. 263851.

[16] Ong, E.B., et al., Vipirinin, a coumarin-based HIV-1 Vpr inhibitor, interacts with a hydrophobic region of VPR. J Biol Chem. 286(16): p. 14049-56.

[17] Pan, R., et al., Anti-arthritic effect of scopoletin, a coumarin compound occurring in Erycibe obtusifolia Benth stems, is associated with decreased angiogenesis in synovium. Fundam Clin Pharmacol. 24(4): p. 477-90.

[18] Bhattacharyya, S.S., et al., Anti-oncogenic potentials of a plant coumarin (7-hydroxy-6-methoxy coumarin) against 7,12dimethylbenz [a] anthracene-induced skin papilloma in mice: the possible role of several key signal proteins. Zhong Xi Yi Jie He Xue Bao. 8(7): p. 645-54.

[19] Bucolo, C., et al., Protective effects of a coumarin derivative in diabetic rats. Invest Ophthalmol Vis Sci, 2009. 50(8): p. 3846-52.

[20] Morris, G.M., et al., AutoDock4 and AutoDockTools4: Automated docking with selective receptor flexibility. J Comput Chem, 2009. 30(16): p. 2785-91.

[21] Trott, O. and A.J. Olson, AutoDock Vina: improving the speed and accuracy of docking with a new scoring function, efficient optimization, and multithreading. J Comput Chem. 31(2): p. 45561.

[22] Mou, T.C., et al., Structural basis for the inhibition of mammalian membrane adenylyl cyclase by 2 '(3')-O-(N-Methylanthraniloyl)guanosine 5 '-triphosphate. J Biol Chem, 2005. 280(8): p. 7253-61.

[23] Sanner, M.F., Python: a programming language for software integration and development. J Mol Graph Model, 1999. 17(1): p. 57-61.

[24] Seeliger, D. and B.L. de Groot, Ligand docking and binding site analysis with PyMOL and Autodock/Vina. J Comput Aided Mol Des. 24(5): p. 417-22.

[25] Park, J.Y., et al., Identification of the peptides that inhibit the stimulation of thyrotropin receptor by Graves' immunoglobulin G from peptide libraries. Endocrinology, 1997. 138(2): p. 617-26.

[26] Brown, B.L., et al., A simple and sensitive saturation assay method for the measurement of adenosine 3':5'-cyclic monophosphate. Biochem J, 1971. 121(3): p. 561-2.

[27] Kim, Y.J., Antimelanogenic and antioxidant properties of gallic acid. Biol Pharm Bull, 2007. 30(6): p. 1052-5.

[28] Yoon, W.J., et al., Effect of palmitoleic acid on melanogenic protein expression in murine b16 melanoma. J Oleo Sci. 59(6): p. 315-9.

[29] Kim, D.C., et al., Inhibition of melanogenesis by 5,7dihydroxyflavone (chrysin) via blocking adenylyl cyclase activity. Biochem Biophys Res Commun.

[30] Lee, Y.S., et al., Inhibitory effect of glyceollin isolated from soybean against melanogenesis in B16 melanoma cells. BMB Rep. 43(7): p. 461-7.

[31] Liu, T., et al., BindingDB: a web-accessible database of experimentally determined protein-ligand binding affinities. Nucleic Acids Res, 2007. 35(Database issue): p. D198-201.

[32] Yano, J.K., et al., Structures of human microsomal cytochrome P450 2A6 complexed with coumarin and methoxsalen. Nat Struct Mol Biol, 2005. 12(9): p. 822-3.

[33] Pandey, K.B. and S.I. Rizvi, Protective effect of resveratrol on markers of oxidative stress in human erythrocytes subjected to in vitro oxidative insult. Phytother Res. 24 Suppl 1: p. S11-4.

[34] Bae, J.Y., et al., Dietary compound ellagic acid alleviates skin wrinkle and inflammation induced by UV-B irradiation. Exp Dermatol. 19(8): p. e182-90.

[35] Domingo, D.S., et al., Anti-angiogenic effects of epigallocatechin3-gallate in human skin. Int J Clin Exp Pathol. 3(7): p. 705-9.

[36] Matsui, Y., et al., Extract of Passion Fruit ( Passiflora edulis ) Seed Containing High Amounts of Piceatannol Inhibits Melanogenesis and Promotes Collagen Synthesis. J Agric Food Chem.

[37] Yokozawa, T. and Y.J. Kim, Piceatannol inhibits melanogenesis by its antioxidative actions. Biol Pharm Bull, 2007. 30(11): p. 2007-11. 